\title{
Impulsivity in abstinent alcohol and polydrug dependence: a multidimensional approach
}

\author{
Eleanor M. Taylor ${ }^{1}$ (D) Anna Murphy ${ }^{1} \cdot$ Venkat Boyapati $^{1} \cdot$ Karen D. Ersche $^{2,3}$. \\ Remy Flechais ${ }^{4} \cdot$ Shankar Kuchibatla $^{1}$. John McGonigle ${ }^{4}$ - Anotonio Metastasio ${ }^{1}$. \\ Liam Nestor ${ }^{4,5} \cdot$ Csaba Orban $^{4} \cdot$ Fillippo Passetti $^{4,2,3} \cdot$ Louise Paterson $^{4}$. \\ Dana Smith $^{2,6}$ • John Suckling ${ }^{2,3} \cdot$ Roger Tait $^{2}$ • Anne R. Lingford-Hughes ${ }^{4}$. \\ Trevor W. Robbins ${ }^{2,6}$ • David J. Nutt ${ }^{4}$. JF William Deakin ${ }^{1} \cdot$ Rebecca Elliott $^{1}$. \\ ICCAM Platform
}

Received: 4 June 2015 / Accepted: 10 February 2016 /Published online: 25 February 2016

(C) The Author(s) 2016. This article is published with open access at Springerlink.com

\begin{abstract}
Rationale Dependence on drugs and alcohol is associated with impaired impulse control, but deficits are rarely compared across individuals dependent on different substances using several measures within a single study.

Objectives We investigated impulsivity in abstinent substance-dependent individuals (AbD) using three complementary techniques: self-report, neuropsychological and neuroimaging. We hypothesised that AbDs would show increased impulsivity across modalities, and that this would depend on length of abstinence.

Methods Data were collected from the ICCAM study: 57 control and 86 AbDs, comprising a group with a history of dependence on alcohol only $(n=27)$ and a group with history of

*ICCAM Platform collaborators David Nutt, Anne Lingford-Hughes, Louise Paterson, John McGonigle, Remy Flechais, Csaba Orban, Bill Deakin, Rebecca Elliott, Anna Murphy, Eleanor Taylor, Trevor Robbins, Karen Ersche, John Suckling, Dana Smith, Laurence Reed, Filippo Passetti, Luca Faravelli, David Erritzoe, Inge Mick, Nicola Kalk, Adam Waldman, Liam Nestor, Shankar Kuchibatla, Venkataramana Boyapati, Antonio Metastasio, Yetunde Faluyi, Emilio Fernandez-Egea, Sanja Abbott, Barbara Sahakian, Valerie Voon, Ilan Rabiner.
\end{abstract}

Electronic supplementary material The online version of this article (doi:10.1007/s00213-016-4245-6) contains supplementary material, which is available to authorized users.

Eleanor M. Taylor

eleanor.taylor-2@manchester.ac.uk

1 Neuroscience and Psychiatry Unit, Institute of Brain Behaviour and Mental Health, University of Manchester, Manchester, UK

2 Behavioural and Clinical Neuroscience Institute, University of Cambridge, Cambridge, UK dependence on multiple substances ("polydrug", $n=59$ ). All participants completed self-report measures of impulsivity: Barratt Impulsiveness Scale, UPPS Impulsive Behaviour Scale, Behaviour Inhibition/Activation System and Obsessive-Compulsive Inventory. They also performed three behavioural tasks: Stop Signal, Intra-Extra Dimensional SetShift and Kirby Delay Discounting; and completed a Go/ NoGo task during fMRI.

Results AbDs scored significantly higher than controls on selfreport measures, but alcohol and polydrug dependent groups did not differ significantly from each other. Polydrug participants had significantly higher discounting scores than both controls and alcohol participants. There were no group differences on the other behavioural measures or on the fMRI measure.
3 Department of Psychiatry, University of Cambridge, Cambridge, UK

4 Centre for Neuropsychopharmacology, Division of Brain Sciences, Imperial College London, London, UK

5 GlaxoSmithKline Clinical Research Unit, Cambridge, UK

6 Department of Psychology, University of Cambridge, Cambridge, UK 
Conclusions The results suggest that the current set of selfreport measures of impulsivity is more sensitive in abstinent individuals than the behavioural or fMRI measures of neuronal activity. This highlights the importance of developing behavioural measures to assess different, more relevant, aspects of impulsivity alongside corresponding cognitive challenges for fMRI.

Keywords Addiction · Alcohol · Cognition · Drug Abuse · FMRI

\section{Introduction}

Impulsivity is action without forethought, involves premature responding, poor response inhibition and low tolerance for delay (Evenden 1999). It is frequently associated with substance dependence (Dalley et al. 2011; de Wit 2009; Perry and Carroll 2008; Verdejo-García et al. 2008) and higher impulsivity is related to polydrug use (McCown 1988; Semple et al. 2005). Although humans use many different substances legally and illegally, heroin, cocaine and alcohol are rated as the most harmful in the UK (Nutt et al. 2010); these were the focus of the present study.

When investigating the link with substance dependence, impulsivity has been measured in many ways, using either self-report questionnaires or behavioural measures (VerdejoGarcía et al. 2008). However, self-report and behavioural measures are rarely correlated (Bari and Robbins 2013; Broos et al. 2012), as each measure looks at distinct attributes, often conceptualised in very different ways. Self-report measures, such as the widely used Barratt Impulsiveness Scale (BIS-11; Patton et al. 1995), are assumed to be relatively stable trait constructs, whilst behavioural measures are dependent on specific strategies that may differ between individuals and testing sessions (Bari and Robbins 2013). Whilst self-report measures may be more ecologically valid, they are reliant on individual insight and are susceptible to bias (Verdejo-García et al. 2008).

Two commonly used cognitive paradigms are the Go/ NoGo Task (GNG), which measures the ability to inhibit a response before it is initiated, and the Stop Signal Task (SST), which measures inhibition of a response after it is initiated. Both tasks have revealed decreased inhibitory control in cocaine dependence (Ersche et al. 2011; Fernández-Serrano et al. 2012; Kaufman et al. 2003) and alcohol dependence (Sjoerds et al. 2014). A recent meta-analysis of these tasks (Smith et al. 2014) found decreased inhibitory control in alcohol and cocaine dependence, but not in opioid dependence. However, there were very few studies using the GNG task in opioid users and none using the SST. One study since has used the SST in opioid dependence, finding increased impulsivity (Liao et al. 2014).
Studies of the neural substrates of impulsivity emphasise the importance of top-down control of subcortical structures, such as the nucleus accumbens (ventral striatum) by frontal brain regions particularly the orbitofrontal cortex (OFC), anterior cingulate cortex (ACC) and dorsolateral prefrontal cortex (DLPFC; Aron et al. 2003; Hester and Garavan 2004; Kaufman et al. 2003). The inferior frontal gyrus (IFG), especially right sided, ACC and DLPFC are frequently implicated in SST and GNG tasks (Chambers et al. 2009; Garavan et al. 2006; Simmonds et al. 2008). Reduced activations associated with poorer inhibitory control in stimulant users have been observed in the $\mathrm{ACC}$ and pre-supplementary motor area (preSMA; Kaufman et al. 2003; Hester and Garavan 2004; Li et al. 2008), as well as the right superior frontal gyrus (Hester and Garavan 2004) and right insula (Kaufman et al. 2003). Reduced prefrontal activation associated with decreased inhibitory control has also been observed in alcohol dependent individuals ( $\mathrm{Li}$ et al. 2009), whilst neuroimaging studies comparing opioid dependent individuals to controls have found performance impairments accompanied by reduced prefrontal, insula and limbic system responses (Forman et al. 2004; Fu et al. 2008).

Recent investigations of impulsivity pay particular attention to the multifaceted nature of the construct and suggest that different forms of impulsivity are influential at different stages of dependence. For example, impulsive choice (measured using delay discounting and Iowa Gambling Task measures) predicts relapse, whilst impulsive action (measured using SST) does not differentiate abstinent and relapsed participants (Stevens et al. 2015). High impulsive choice is associated with continued drug use and poor maintenance of abstinence (Passetti et al. 2008; MacKillop and Kahler 2009; Washio et al. 2011; Stevens et al. 2013; Stevens et al. 2014), whilst impulsive action is thought to be related to initial sensitivity (Diergaarde et al. 2008; Broos et al. 2012). Stevens et al. (2015) also suggest that behavioural measures are more useful than trait measures for detecting relapse risk, and imply that the different types of measures may be more useful at the different stages of addiction.

A key question is the extent to which impulsivity varies with abstinence. There is evidence for recovery of executive functioning during abstinence (Sullivan et al. 2000; Schulte et al. 2014; Stavro et al. 2013; Fernández-Serrano et al. 2011), as well as more specific evidence for normalisation of behavioural inhibitory control (Hopwood et al. 2011; Morie et al. 2014; Bell et al. 2014). However, findings are not consistent, with other studies reporting that behavioural impulsivity is still elevated in abstinent alcohol dependent participants with mean length of abstinence of six months (Naim-Feil et al. 2014). It has been suggested that abstinence exceeding 12 months may be 
critical in terms of recovery, as $65-75 \%$ of AbDs relapse within 12 months of treatment discharge (Sinha 2011). Poor treatment retention and early relapse are associated with higher impulsivity in dependence (Moeller et al. 2001; Patkar et al. 2004; Evren et al. 2012) suggesting that impulsivity mechanisms may be a determinant of sustained abstinence, such that impulsivity measures may be different in those who are able to maintain abstinence longer. There is therefore a need for studies that systematically examine variability in impulsivity associated with varying length of abstinence (extending beyond 12 months).

The evidence for impulsivity in substance dependence is not consistent, especially when we consider the different types of substance dependence (for a review, see Smith et al. 2014). However, there has been little research comparing groups with different dependencies within a single study and the majority of papers have fewer than 30 participants per group (Smith et al. 2014), only providing sufficient power to detect moderate effect sizes in questionnaire and behavioural measures. Therefore, the aim of the present study was to investigate impulsivity measures across different modalities: self-report, behavioural and neuroimaging, in a large number of abstinent participants with a history of dependence on different substances. Although humans use many different substances legally and illegally, heroin, cocaine and alcohol are rated as the most harmful in the UK (Nutt et al. 2010); these were the focus of the present study. We hypothesised that AbDs would show increased impulsivity across all modalities and that this would be more marked in those with dependence on multiple substances compared to those dependent on alcohol alone. A secondary aim was to explore how impulsivity measures vary with length of abstinence, extending to beyond 12 months.

\section{Methods}

This study was conducted as part of the ICCAM Platform Study (www.iccam.org.uk), details of which are reported by Paterson et al. (2015). The protocol was approved by the West London Research Ethics Committee (REC Ref: 11/H0707/9; PI: Prof D.J. Nutt). Non-imaging testing sessions were conducted at three sites: NIHR/Wellcome Trust Imperial Clinical Research Facility, NIHR/Wellcome Trust Cambridge Clinical Research Facility, and Clinical Trials Unit, Salford Royal NHS Foundation Trust. Imaging sessions were conducted in the adjoining centres at Imanova Limited (formerly the GSK Clinical Imaging Centre), Wolfson Brain Imaging Centre, Manchester Translational Imaging Unit (3T MRI Facility), respectively.

\section{Participants}

Participants, including abstinent substance-dependent individuals (AbDs) and controls, were recruited from local NHS addiction services and via advertising on social media, in job centres and libraries. Following written and informed consent, all participants were assessed using the Structured Clinical Interview for DSM-IV to assess for dependence history and checked by a psychiatrist. Exclusion criteria for all participants included lifetime history of psychotic disorder, neurological illness, neurodevelopmental disorder or traumatic head injury. Participants were between 20-65 years old and able to read and write in English. To confirm abstinence on day of testing, all participants completed an alcohol breath test and urine drug screen. Participants were requested to refrain from cannabis use for at least seven days prior to each session but, given the long half-life of cannabinoid metabolites, positive results for cannabinoids were permitted if the participant was not intoxicated or in withdrawal (determined by the psychiatrist conducting the interview). AbDs were abstinent for at least two weeks prior to testing. Nicotine use was not an exclusion criterion in any group as the majority of substancedependent individuals smoke tobacco.

Of the 179 participants who were consented to the study across the three sites, 14 were excluded immediately at initial screening by the researcher on the basis of obviously failing to meet inclusion criteria (e.g. a history of psychosis). A further 22 participants were subsequently excluded by the clinical committee at a second reviewing stage on the basis of more subtle exclusions (e.g. controls with past cannabis use felt to be approaching dependent levels). This left 143 who were eligible for inclusion in analyses $(21.7 \%$ female, aged 25 64, mean 41.66, SD 8.93) comprising 57 control participants with no history of substance dependence (except nicotine), and 86 abstinent substance-dependent participants (see Table 1). The majority of substance-dependent participants had experience with a large number of substances and many met criteria for past dependence on multiple substances. For the purposes of this study, we defined two substance-dependent groups: "alcohol AbD" participants who met DSM-IV criteria for past dependence on alcohol $(n=27)$ and "polydrug AbD" participants who met DSM-IV criteria for past dependence on two or more substances, one of which was alcohol, cocaine or heroin ( $n=59$; see Table 2$)$.

Three control participants did not complete the behavioural tasks, leaving 54 control participants in the behavioural analysis. Fourteen participants were removed from the GNG imaging analysis due to excessive movement (defined as $>20 \%$ volumes with $>1 \mathrm{~mm}$ movement) or low baseline performance on the GNG imaging task ( $<85 \%$ Go accuracy), leaving 52 control, 26 alcohol and 51 polydrug participants in the imaging analysis. 
Table 1 Demographic data for control, alcohol dependent and polydrug dependent participants

\begin{tabular}{llllrll}
\hline & Control & Alcohol & Polydrug & F or $\chi^{2}$ & df & $p$ \\
\hline Age (mean, SD) & $42.39(8.74)$ & $45.81(8.49)$ & $39.05(8.57)$ & 6.034 & 2,140 & 0.003 \\
IQ (mean, SD) & $107.88(8.83)$ & $104.85(7.59)$ & $99.32(10.86)$ & 11.904 & 2,140 & $<0.001$ \\
\% Female & 26.3 & 22.2 & 16.9 & 1.504 & 2 & 0.471 \\
\% Smokers & 56.1 & 74.1 & 76.3 & 5.979 & 2 & 0.050 \\
\hline
\end{tabular}

\section{Assessment procedure}

\section{Clinical variables}

Participants were interviewed to ascertain eligibility and group allocation. We also obtained data on their substances of dependence (excluding nicotine) and length of abstinence (see Table 2). For alcohol AbD participants, length of abstinence was calculated from their last use of alcohol to dependent levels. For polydrug $\mathrm{AbD}$ participants the multiple substances meant that length of abstinence could only be calculated from the most recent use of any substance of dependence.

\section{Self-report questionnaires}

Participants completed a battery of impulsivity questionnaires presented in computer format. These included the Barratt Impulsiveness Scale (BIS-11; Patton et al. 1995), Behaviour Inhibition/Activation System (BIS/BAS; Carver and White 1994), the UPPS Impulsive Behaviour Scale (UPPS-P; Whiteside and Lynam 2003) and the Obsessive-Compulsive Inventory Revised (OCI-R; Foa et al. 2002).

\section{Behavioural tasks}

The Kirby test of delay discounting (Kirby and Maraković 1996) measures the discounting rate; the extent to which the present value of a future reward decreases as the delay to its receipt increases. Hypothetical immediate rewards of $£ 11-80$ and delayed rewards of $£ 25-85$, with delays of 7-186 days were used. A hyperbolic discount parameter $(k)$ score for each participant was generated from the proportion of immediate choices that were made over delayed choices using the method reported by Kirby et al. (1999); and Kirby (2000). Greater discounting, indexed by increasing $k$ values, indicates higher levels of impulsivity.

Participants also completed the Stop Signal Task (SST) and the Intra-Extra Dimensional Set Shift (IED) task from the well-validated CANTAB neuropsychological test battery (www.cambridgecognition.com/academic/cantabsuite/ executive-function-tests). The SST is a test of motor inhibition, specifically action cancellation (Dalley et al. 2011), at the presentation of an auditory stimulus. A full description is presented by Ersche and Sahakian (2007). The primary outcome is the "stop-signal reaction time" (SSRT), which is the time an individual requires to withhold a response.

The IED is derived from the Wisconsin Card Sorting Task and assesses rule acquisition and reversal, visual discrimination, attentional set formation, maintenance, shifting and flexibility of attention. Primary outcome measures are "total errors" (adjusted for any early terminations), "number of stages completed" and "number of errors at each stage". A full description is presented in Downes et al. (1989).

\section{Functional MR imaging tasks}

To investigate neural substrates of inhibitory control, participants performed a Go/NoGo (GNG) task whilst being scanned using fMRI (Fig. 1). Participants were presented with a series of individual "X's" and "Y's" and asked to respond as quickly
Table 2 Substance dependence data for control, alcohol dependent and polydrug dependent participant groups.

Data exclude nicotine

dependence

\begin{tabular}{llllll}
\hline & & Control & Alcohol & Polydrug & Total \\
Alcohol dependence & No & 57 & 0 & 23 & 80 \\
& Yes & 0 & 27 & 36 & 63 \\
Cocaine dependence & No & 57 & 27 & 14 & 98 \\
& Yes & 0 & 0 & 45 & 45 \\
Opioid dependence & No & 57 & 27 & 20 & 104 \\
& Yes & 0 & 0 & 39 & 39 \\
Length of abstinence (months) & Alcohol & Polydrug & Polydrug (outlier removed) \\
Mean (SD) & $15.46(17.76)$ & $20.86(43.98)$ & 15.63 (18.10) \\
Median & 8.0 & 9.0 & & 8.5 \\
Range & $1.0-79.0$ & $0.5-324.0$ & $0.5-102.0$ \\
\hline
\end{tabular}




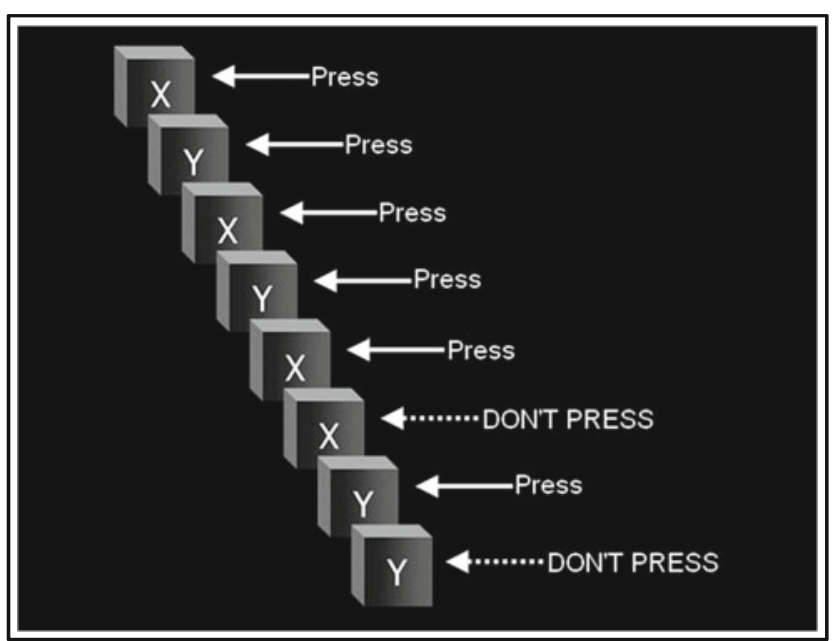

Fig. 1 An instruction screen from the Go/NoGo task. Participants are asked to respond as quickly as possible to each letter " $\mathrm{X}$ " and " $Y$ " that appears on the screen, except when the letter is the same as the one shown previously

as possible to each letter by pressing a button (Go), except when it immediately repeated itself (NoGo). This was an event-related task carried out in two runs of 250 trials, each containing 220 Go trials and 30 NoGo trials. This ratio of frequent Go to rare NoGo trials was used as it is considered a stronger test of pure inhibition than other Go:NoGo ratios (Smith et al. 2014). Each letter was presented for $900 \mathrm{~ms}$ and followed by $100 \mathrm{~ms}$ inter-stimulus interval of a blank screen. Each run began with a 12-s fixation and lasted for $262 \mathrm{~s}$. Immediately before scanning, participants completed 60 practice trials.

\section{MR image acquisition}

Imaging was carried out at the three sites using a Siemens (Imperial and Cambridge) or a Philips (Manchester) 3T MR scanner. One hundred and thirty one volumes were acquired, comprising 33-36 axial slices of $3 \mathrm{~mm}$ thickness, with a TR of $2000 \mathrm{~ms}$, TE of $32 \mathrm{~ms}$ and a voxel size of $3 \times 3 \times 3 \mathrm{~mm}$. In order to maximise cerebral coverage whilst minimising slice thickness and susceptibility artefact, fMRI/EPI acquisition was at +30 degrees to the ACPC line. A T1-weighted structural image was also acquired for use in spatial pre-processing and for examination of any structural abnormalities.

\section{Data analysis}

\section{Self-report and behavioural data analysis}

Data were analysed using Statistical Package for Social Sciences (SPSS, version 22, www.spss.com) firstly using multivariate analysis of variance (MANOVA) to asses overall group differences. Where significant main effects were found, these were explored with individual univariate analyses of variance, and finally Tukey's LSD post hoc test when main effects from the univariate analyses were found. Pearson's Chi-square tests or independent $t$ tests were used to assess group differences in demographic variables. In order to explore important relationships between impulsivity measures and length of abstinence, Pearson's correlations were used within the AbD groups. We also used Pearson's correlation to explore whether any of the measures where there were significant group differences were related to age, IQ or smoking status in the $\mathrm{AbD}$ groups.

\section{Image analysis}

Imaging data were analysed using statistical parametric mapping (SPM12; Wellcome Trust Centre for Neuroimaging, London, England, http://www.fil.ion.ucl.ac.uk/spm/), implemented in MATLAB (Mathworks 2012, www. mathworks.com). Images were realigned to correct for motion, using the first image as a reference. The structural (T1-weighted) and functional images were then coregistered, followed by spatial normalisation, and were smoothed using a Gaussian kernel filter of $8 \times 8 \times 8 \mathrm{~mm}$. First level analysis was performed on the contrasts of "Stops" (successful inhibitions) compared to a background of Go responding. Errors of commission were modelled as a contrast of no interest as there were too few for sufficient power. Also modelled as contrasts of no interest were "Sleep", where there were more than 10 consecutive errors of omission on Go trials, and "False Inhibitions" where successful inhibitions on NoGo trials were immediately preceded by an omitted Go trial.

The second level analysis used a region of interest (ROI) approach based on areas previously identified in the inhibitory control literature as being altered in substance dependence. The areas identified were the right and left inferior frontal gyri (IFG) and the anterior cingulate cortex (ACC), defined by Neuromorphometrics, Inc. (www.neuromorphometrics.com), under academic subscription (Supplementary Figure 1). We extracted the average value of the Stops contrast per person within each ROI and performed group comparisons using independent-samples $t$ tests in SPSS. Correlation analyses were conducted by performing one-way ANOVAs with the variables of "NoGo accuracy" and "length of abstinence" entered as separate covariates $(p<0.05$, Bonferroni corrected for three comparisons). To investigate the relationship with length of abstinence, one polydrug participant was removed due to an outlying length of abstinence score (Table 2) and correlations between BOLD signal in ROIs and length of abstinence were then examined in the alcohol and polydrug $\mathrm{AbD}$ groups. 


\section{Results}

\section{Demographics and clinical variables}

Participant group demographics can be seen in Table 1, whilst additional dependencies and length of abstinence can be seen in Table 2. One polydrug AbD participant was removed from the length of abstinence analysis due to an outlying length of abstinence of more than two standard deviations from the mean. There were no differences in length of abstinence between alcohol and polydrug AbD participants $(\mathrm{t}(83)=-0.040$, $p=0.968)$.

\section{Normalisation of data}

Initial data screening using Q-Q plots highlighted nonnormally distributed scores for IED total errors, Kirby and OCI-R. Therefore, these data were transformed using a log transformation, with the resulting Q-Q regressor plots showing normal distribution.

\section{Self-report measures}

\section{Multivariate analysis: group differences on total scores}

The effect of group (control, alcohol AbD, polydrug $\mathrm{AbD}$ ) was analysed using a multivariate analysis of variance (MANOVA) conducted on total scores for each of the self-report measures of BIS-11, UPPS-P, BIS/BAS and OCI-R. Using Pillai's trace, a significant main effect of group was found $(V=0.395, \mathrm{~F}(10,274)=6.741$, $p<0.001)$.

This significant main effect allowed separate univariate analyses of variance (ANOVA) to be performed post hoc on each of the outcome variables. These revealed significant group differences on the total scores of BIS-11 $(\mathrm{F}(2,140)=22.676, p<0.001)$, UPPS-P $(\mathrm{F}(2,140)=39.284$, $p<0.001)$, BAS $(\mathrm{F}(2,140)=6.319, p<0.01)$ and OCI-R $(\mathrm{F}(2,140)=6.123, p<0.01)$. There was no main effect of group on BIS total $(\mathrm{F}(2,140)=1.414, p=0.247)$. Further post hoc analysis using Tukey's LSD revealed that both alcohol and polydrug $\mathrm{AbD}$ groups scored significantly higher on the BIS-11 (alcohol $p<0.01$; polydrug $p<0.001$ ), UPPS-P (alcohol $p<0.001$; polydrug $p<0.001$ ) and OCI-R (alcohol $p<0.05$; polydrug $p<0.01)$ total scores than controls, whilst only the polydrug AbD group scored significantly higher than controls on $\operatorname{BAS}(p<0.01)$ total scores (Fig. 2 and Table 3).

\section{Group differences on sub-scores}

A second MANOVA was conducted to assess for group differences on each of the questionnaire sub-scores. These included the BIS-11 (Attentional Impulsivity, Motor Impulsivity, Non-Planning Impulsivity), UPPS-P (Negative Urgency, Premeditation, Perseverance, Sensation Seeking, Positive Urgency), and BIS/BAS (Drive, Fun, Reward). Using Pillai's trace, a significant main effect of group was found $(V=0.567, \mathrm{~F}(22,262)=4.712, p<0.001)$, allowing for separate univariate ANOVAs to be performed.

These post hoc ANOVAs revealed a significant group difference on BIS-11 Attentional Impulsivity $(\mathrm{F}(2,140)=19.128$, $p<0.001)$, Motor Impulsivity $(\mathrm{F}(2,140)=8.751, p<0.001)$, and Non-Planning Impulsivity $(\mathrm{F}(2,140)=22.801$, $p<0.001)$; UPPS-P Negative Urgency $(\mathrm{F}(2,140)=35.125$, $p<0.001)$, Premeditation $(\mathrm{F}(2,140)=7.521, p<0.01)$, Perseverance $(\mathrm{F}(2,140)=16.818, p<0.001)$, Sensation Seeking $(\mathrm{F}(2,140)=6.882, p<0.01)$, and Positive Urgency $(\mathrm{F}(2,140)=26.695, p<0.001)$; BIS/BAS Drive $(\mathrm{F}(2$, $140)=5.249, p<0.01)$ and BIS/BAS Fun $(\mathrm{F}(2,140)=6.071$, $p<0.01)$. There was no main effect of group on BIS/BAS Reward $(\mathrm{F}(2,140)=1.766, p=0.175)$.

Further post hoc analysis using Tukey's LSD revealed that both alcohol and polydrug $\mathrm{AbD}$ groups scored significantly higher than controls on BIS-11 Attentional Impulsivity (alcohol $p<0.001$; polydrug $p<0.001$ ), Motor Impulsivity (alcohol $p<0.05$; polydrug $p<0.001$ ), and Non-Planning Impulsivity (alcohol $p<0.05$; polydrug $p<0.001$ ), whilst polydrug participants also scored significantly higher than alcohol participants on Non-Planning Impulsivity (alcohol $p<0.05$ ). Both alcohol and polydrug AbD groups scored significantly higher than controls on UPPS-P Negative Urgency (alcohol $p<0.001$; polydrug $p<0.001$ ), Perseverance (alcohol $p<0.01$; polydrug $p<0.001$ ), and Positive Urgency (alcohol $p<0.001$; polydrug $p<0.001)$. Polydrug AbD participants scored significantly higher than controls on UPPS-P Premeditation $(p<0.001)$, BIS/BAS Drive $(p<0.01)$ and BIS/BAS Fun $(p<0.01)$, as well as higher than both controls and alcohol AbD participants on UPPS-P Sensation Seeking (control $p<0.01$; alcohol $p<0.05$ ).

\section{Behavioural tasks}

The effect of group (control, alcohol AbD, polydrug AbD) was analysed using a MANOVA on the score outcome measures for each of the behavioural tasks; SSRT, IED total errors and Kirby $k$ (Table 4). Using Pillai's trace, a significant main effect of group was found $(V=0.123, \mathrm{~F}(3,272)=2.978$, $p<0.01$, allowing for separate univariate ANOVAs to be performed. These post hoc ANOVAs revealed a significant group difference on Kirby $k(\mathrm{~F}(2,137)=6.244, p<0.01)$, but no significant group differences on $\operatorname{SSRT}(\mathrm{F}(2,137)=0.822$, $p=0.442)$ or IED total errors $(\mathrm{F}(2,137)=2.402, p=0.094)$. Further post hoc analysis using Tukey's LSD revealed that polydrug participants had significantly higher discounting scores than controls $(p<0.01)$, whilst those of alcohol 
Fig. 2 Total scores on self-report measures for alcohol and polydrug $\mathrm{AbD}$ groups plotted as their difference from control scores. ${ }^{*} p<0.05, * * p<0.01$, $* * * p<0.001$

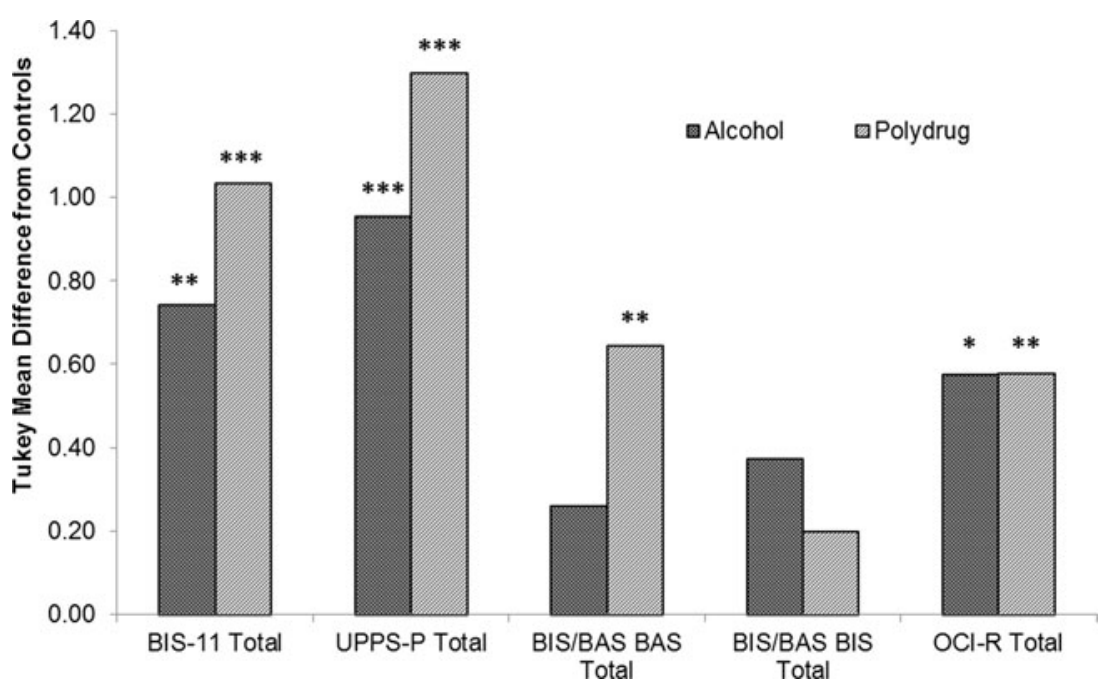

participants were only marginally higher than controls $(p=0.054)$.

Additional exploratory analysis was performed on further outcome measures of the SST and IED in order to ensure these were not confounding the results. All participants did not differ on Stop Signal Delay $(\mathrm{F}(2,137)=0.369, p=0.692)$, Successful Stops $(\mathrm{F}(2,137)=1.292, p=0.278)$, or mean Go Reaction Times $(\mathrm{F}(2,137)=0.203, p=0.816)$. There were no significant group differences in the number of IED stages completed $\left(\chi^{2}=10.321, p=0.413\right)$ nor number of errors at each stage.

\section{Length of abstinence}

In each of the $\mathrm{AbD}$ groups, we conducted correlation analyses on the measures where significant differences from controls were observed to explore how these measures related to length of abstinence (Table 5). Shorter length of abstinence was associated with higher scores on BIS-11 Non-Planning Impulsivity $(r=-0.44, p<0.05)$, UPPS Negative Urgency $(r=-0.41, p<0.05)$ and Premeditation $(r=-0.38, p<0.05)$, as well as on BIS/BAS Fun $(r=-0.38, p<0.05)$ within the alcohol AbD group. Higher Kirby scores only were found to be associated with shorter length of abstinence in the polydrug AbD group $(r=-0.40, p<0.01)$.

\section{Correlation analyses associations with age, IQ and smoking status}

Correlation analyses were also conducted to assess whether the variables of age, IQ and smoking status might explain the variance in impulsivity measures across groups. We conducted analyses separately within the alcohol and polydrug AbD groups on impulsivity measures where the groups differed significantly from controls. There were no significant correlations (see Supplementary Table 1).

\section{FMRI analysis}

An ANOVA showed no significant group difference in Go RT $(\mathrm{F}(2,126)=1.045, p=0.355)$ or NoGo accuracy $(\mathrm{F}(2$, $126)=0.985, p=0.376)$. There was, however, a significant difference in Go accuracy $(\mathrm{F}(2,126)=4.402, p<0.05)$. Key values are summarised in Table 6 .

Independent $t$ tests between alcohol dependent vs control, polydrug dependent vs control, and alcohol dependent vs polydrug dependent, did not identify any significant BOLD signal changes at the voxel-level Family Wise Error (FWE) corrected threshold of $p<0.05$. We also used the predefined ROIs of right IFG, left IFG and ACC, none of which identified any significant group differences. Correlation analyses found no significant associations between activation and either NoGo performance or length of abstinence.
Table 3 Mean total scores (and $\mathrm{SD})$ for control, alcohol $\mathrm{AbD}$ and polydrug $\mathrm{AbD}$ participants on each of the self-report measures

\begin{tabular}{lrlrlrl}
\hline & Control & \multicolumn{3}{c}{ Alcohol } & \multicolumn{3}{c}{ Polydrug } \\
\hline BIS-11 total & 56.88 & $(9.45)$ & 66.59 & $(12.63)$ & 70.41 & $(11.58)$ \\
UPPS_P total & 112.70 & $(19.27)$ & 138.15 & $(24.72)$ & 147.32 & $(21.83)$ \\
BIS/BAS BAS total & 37.35 & $(5.68)$ & 38.78 & $(6.01)$ & 40.88 & $(4.71)$ \\
BIS/BAS BIS total & 19.09 & $(4.28)$ & 20.67 & $(3.96)$ & 19.93 & $(4.20)$ \\
OCI total & 7.40 & $(7.93)$ & 13.59 & $(13.43)$ & 13.02 & $(10.05)$ \\
\hline
\end{tabular}


Table 4 Mean (SD) scores for each of control, alcohol and polydrug groups on the three behavioural measures of impulsivity

\begin{tabular}{lrlclcl}
\hline & Control & & \multicolumn{2}{c}{ Alcohol dependent } & \multicolumn{2}{c}{ Polydrug dependent } \\
\hline SSRT & 183.61 & $(46.73)$ & 190.98 & $(48.51)$ & 195.31 & $(50.47)$ \\
IED errors (adj.) & 26.11 & $(28.56)$ & 34.52 & $(36.93)$ & 34.19 & $(29.77)$ \\
Kirby $k$ & 0.02 & $(0.02)$ & 0.04 & $(0.05)$ & 0.04 & $(0.06)$ \\
\hline
\end{tabular}

\section{Effects of different dependency histories}

Whilst numbers were too small for definitive analysis, we performed an exploratory MANOVA of the 59 polydrug AbD participants to determine whether differences could be attributed to a specific history of dependence on the most commonly used substances in our sample (alcohol, opioids or stimulants). Using Pillai's trace, a significant main effect of past stimulant dependence was found on total self-report scores $(V=0.189$, $\mathrm{F}(5,53)=2.463, p<0.05)$, which was found to be due to those with a history of stimulant dependence scoring significantly higher than those with no history of stimulant dependence on the total scores of BIS-11 ( $\mathrm{F}(1$, $57)=6.171, p<0.05)$, UPPS -P $(\mathrm{F}(1,57)=7.596$, $p<0.01)$, BIS $(\mathrm{F}(1,57)=5.886, p<0.05)$ and OCI-R $(\mathrm{F}(1,57)=8.162, p<0.01)$, but not on BAS $(\mathrm{F}(1,57)=0.109$, $p=0.742)$. There were no differential effects in those with and without opioid use or alcohol use, and no effects of any of the substances on behavioural or imaging measures.

Table 5 Correlation matrix for length of abstinence with measures of impulsivity

\begin{tabular}{llll}
\hline & & Alcohol dependent & Polydrug dependent \\
\hline BIS-11 & Total & -0.344 & -0.066 \\
& Attention & -0.232 & -0.007 \\
& Motor & -0.226 & -0.09 \\
& Non-planning & $-0.436^{*}$ & 0.054 \\
UPPS-P & Total & $-0.455^{*}$ & -0.198 \\
& Negative urgency & $-0.413^{*}$ & -0.188 \\
& Premeditation & $-0.381^{*}$ & -0.161 \\
& Perseverance & -0.349 & -0.086 \\
& Sensation seeking & -0.044 & -0.124 \\
& Positive urgency & -0.368 & -0.123 \\
BIS/BAS & Drive & -0.257 & 0.006 \\
& Fun & $-0.382^{*}$ & -0.137 \\
& BAS total & -0.344 & -0.078 \\
OCI-R & Total & 0.285 & -0.010 \\
Kirby & $k$ & -0.019 & $-0.398 * *$ \\
\hline
\end{tabular}

${ }^{*} p<0.05,{ }^{* *} p<0.01$ (2-tailed)

\section{Discussion}

This investigation compared self-report, behavioural and neural measures of impulsivity in a large abstinent substancedependent $(\mathrm{AbD})$ population. We found that $\mathrm{AbD}$ participants scored higher than controls on most self-report measures, but that alcohol and polydrug dependent groups did not differ from each other. In contrast, there were no group differences on the fMRI GNG task or any of the behavioural measures of impulsivity, except for the Kirby task. These findings add to the growing literature on impulsivity in substance dependence and point to the need for more appropriate and relevant behavioural and neural impulsivity measures for $\mathrm{AbD}$ individuals.

\section{Self-report impulsivity}

Both alcohol and polydrug $\mathrm{AbD}$ groups were found to be more impulsive than controls across all self-report measures, except the Behaviour Inhibition Scale, in line with previous literature (Ersche et al. 2010, 2011; von Diemen et al. 2008). Although the alcohol and polydrug $\mathrm{AbD}$ group scores were not significantly different, there was a trend for the polydrug group to score higher than the alcohol group, perhaps reflecting multiple dependencies (McCown 1988; Semple et al. 2005).

The same pattern was also observed in the subscales of the self-report measures, with both $\mathrm{AbD}$ groups scoring significantly higher than control participants whilst not differing from each other. There was one notable exception, with polydrug $\mathrm{AbD}$ participants reporting more sensation seeking than both controls and alcohol participants (who did not differ from each other). This may be a result of prevalent stimulant dependence history in the polydrug compared to the alcohol AbD group. Stimulants such as cocaine and amphetamines produce alterations in the mesolimbic dopaminergic system (Volkow et al. 2011), which are associated with trait impulsivity (Dalley et al. 2011). Sensation seeking is particularly associated with stimulant use (Ersche et al. 2010; Mahoney et al. 2015), and the higher scores in polydrug AbD participants in our study could be consistent with their higher levels of stimulant use. This is supported by our exploratory post hoc analysis that divided polydrug AbD participants by their specific past dependencies, in which we found that only when stimulant dependence history, and not opioid or alcohol dependence 
Table 6 Mean (SD) performance scores for each group on GNG fMRI task

\begin{tabular}{lrlclcl}
\hline & Control & & \multicolumn{2}{c}{ Alcohol dependent } & \multicolumn{2}{c}{ Polydrug dependent } \\
\hline Go accuracy (\%) & 98.58 & $(2.15)$ & 97.68 & $(2.71)$ & 96.79 & $(3.91)$ \\
NoGo accuracy (\%) & 69.49 & $(15.58)$ & 65.24 & $(17.65)$ & 65.05 & $(18.92)$ \\
Go RT (ms) & 340.18 & $(72.10)$ & 318.16 & $(64.75)$ & 341.64 & $(74.89)$ \\
\hline
\end{tabular}

history, was used as a grouping variable was there a significant difference on self-reported impulsivity. Whilst this suggests that stimulant dependence may be a major determinant of the increased impulsivity in our polydrug $\mathrm{AbD}$ group, these findings should be taken with extreme caution due to the unbalanced groups and low power of this analysis.

Although sensation seeking has been highly related to substance dependence (Zuckerman 2007), there is relatively little evidence linking it directly to alcohol dependence (Noël et al. 2011). This is particularly notable in comparison to the wealth of evidence of elevated sensation seeking in stimulant dependence (e.g. Marusich et al. 2011; Mahoney et al. 2015; Ersche et al. 2010; Stoops et al. 2007; Kelly et al. 2006; Patkar et al. 2004). However, the literature seems to focus more on sensation seeking as a risk factor for heavy drinking in adolescence (e.g. Comeau et al. 2001; Shin et al. 2012; Gillespie et al. 2012). Additionally, impulsivity (specifically sensation seeking and lack of premeditation) were found to be more related to illicit substance use in young adults than was hazardous drinking (Shin et al. 2013). To the best of our knowledge there has not been an investigation comparing sensation seeking in stimulant and alcohol dependence (either current or past) within one study.

\section{Behavioural and neural measures of impulsivity}

There were no group differences on neural measures of impulsivity, nor any of the behavioural measures except for the Kirby delay discounting task. One explanation would be that most of our behavioural and neural measures assess state impulsivity, which undergoes change during and immediately after dependence, whilst self-report measures (together with the Kirby task) assess trait impulsivity that is relatively impervious to such changes. Whilst this conclusion is intuitively appealing, it is at odds with evidence of increased SST impulsivity in siblings of stimulant dependent individuals (Ersche et al. 2011) implicating behavioural impulsivity as an endophenotypic trait. It is also important to note that in the present study, length of abstinence was related to BIS-11 NonPlanning Impulsivity, UPPS-P Negative Urgency and Premeditation, as well as BIS/BAS Fun within the alcohol $\mathrm{AbD}$ group. Kirby discounting scores were also seen to decrease with length of abstinence within the polydrug AbD group. Thus self-reported (and Kirby discounting) impulsivity appear to decrease with extended abstinence, a pattern not entirely consistent with the hypothesis that self-reported impulsivity is a stable trait. However, the pattern we observed could also be explained by lower levels of trait impulsivity in those able to maintain longer term abstinence. A longitudinal approach would be required to distinguish these possibilities. Another consideration is that self-report measures of impulsivity are more susceptible to bias (Verdejo-García et al. 2008), intentional or otherwise, whilst behavioural tasks are much less prone to this bias. Therefore, this begs the question of whether this bias is driving the self-report impulsivity differences rather than "true impulsivity". This may be particularly pertinent for abstinent individuals who have learned to inhibit potentially instinctive impulsive behaviours to maintain abstinence.

Of the explicit behavioural measures used here, only the Kirby discounting scores were seen to decrease with extended abstinence within the polydrug dependent group. Another interpretation is that the SST, IED and GNG simply do not capture the most relevant aspects of impulsivity in AbDs, whilst the self-report measures used in this study (as well as the Kirby) are more sensitive to detecting differences. SST, GNG and, to a lesser extent, IED measure inhibition of motor responses and none of these tasks include emotional or motivational components. By contrast, many of the self-report scales for which we found significant group differences measure how an individual reacts to emotional states, such as the UPPS-P Negative Urgency subscale, which is sensitive to differences between dependent individuals (Torres et al. 2013) as well as pathological gamblers (Clark et al. 2012). Similarly, the Kirby behavioural task involves the motivationally salient/ emotionally relevant cue of money. Although "cold cognitive" tests such as the GNG, SST and IED used here are used widely in the addiction literature, and have been found to be sensitive to current and very recent dependence, it may be that more "hot cognitive" tests, that include emotional and motivational dimensions, are required to detect differences in groups able to maintain abstinence.

Therefore, there is a need for validated behavioural measures that assess "affective impulsivity", in a way that incorporates motivational and emotional components known to be important in both developing substance dependence (Andersen and Teicher 2009; Woicik et al. 2009), and later relapse (Koob and Le Moal 2001). Thus impulsivity tests like the Kirby that involve decision-making about motivationally salient cues may also prove more sensitive than simple motor 
impulsivity tasks. The multidimensional nature of impulsivity (Evenden 1999; Bari and Robbins 2013) suggests that multiple tasks are required to assess the construct comprehensively and our findings highlight the need for more extensive cognitive and behavioural impulsivity assessment in AbDs.

\section{Length of abstinence}

It is also important to note that in the present study, length of abstinence was related to BIS-11 Non-Planning Impulsivity, UPPS-P Negative Urgency and Premeditation, as well as BIS/ BAS Fun within the alcohol AbD group. Kirby discounting scores were also negatively correlated with length of abstinence within the polydrug AbD group. Thus self-reported (and Kirby discounting) impulsivity appears to decrease with extended abstinence, a pattern not entirely consistent with the hypothesis that self-reported impulsivity is a stable trait. However, the pattern we observed could also be explained by lower levels of trait impulsivity in those able to maintain longer term abstinence. Unfortunately, the nature of the design of this study is such that we cannot determine whether impulsivity predated or is a consequence the individuals' drug use. A longitudinal approach would be required to distinguish these possibilities.

Nevertheless, length of abstinence is an important point of variation between previous studies in this area. Ersche et al.'s (2011) stimulant dependent individuals who showed increased impulsivity were not abstinent. Recently Naim-Feil et al. (2014) reported persisting impulsivity in abstinent alcohol dependent participants with a mean duration of abstinence of approximately 6 months. In our sample, mean length of abstinence was over 12 months and may have allowed individuals time for significant cognitive improvement. Although there is some evidence of improvement of inhibitory control with abstinence (Hopwood et al. 2011; Morie et al. 2014), including functioning of relevant neural circuits (Bell et al. 2014), there is also evidence of an improvement in executive functioning (Schulte et al. 2014; Stavro et al. 2013; Sullivan et al. 2000), into which inhibitory control can be included as a wider construct (see also a review by Fernández-Serrano et al. 2011). Consistent with the idea of recovery of executive functions, including inhibitory control, we found less impulsive performance on the Kirby task to be associated with longer abstinence in the polydrug $\mathrm{AbD}$ group.

Alternatively, including only stably abstinent participants may have biased our sample towards individuals with lower cognitive impulsivity, since the most impulsive individuals prone to early relapse will have been excluded. Early relapse and poor treatment retention are associated with higher impulsivity in stimulant dependence, (Moeller et al. 2001; Patkar et al. 2004) and alcohol dependence (Evren et al. 2012), although not in opioid dependence (Passetti et al. 2008). Nevertheless, the correlations between self-report measures and length of abstinence were relatively weak and do not explain all of the variance. Self-reported impulsivity is thus elevated even in those with long-term abstinence.

\section{Limitations}

A limitation of this study is that the polydrug $\mathrm{AbD}$ participants had past dependence on a wide range of substances with substantially different individual profiles of dependent and nondependent use. Whilst the cohort was representative of the substance-dependent population in the UK, this heterogeneity precluded systematic analysis of the contribution of different substances to the effects observed. For example, the specific contribution of a history of stimulant dependence to impulsivity was impossible to isolate. Poly-substance use is an important issue in addiction research, particularly the potential distinction of dependence on stimulants from other substances, with some studies suggesting that opioid dependence is behaviourally distinct from stimulant dependence (Badiani et al. 2011; Vassileva et al. 2014). Thus, for example, high impulsivity in opioid use has been suggested to be a result of drug use rather than a risk factor in the development of dependence (Harty et al. 2011; Schippers et al. 2012), by contrast to stimulant dependence where high impulsivity is a well-established risk factor (Dalley et al. 2011; Ersche et al. 2010). In addition, a recent paper by Whelan et al. (2014) showed that impulsivity played a relatively minor role in the development of alcohol dependence. However, the clinical reality of drug addiction in the UK is a very high prevalence of poly-substance use and dependence; a meta-analysis by Smith et al. (2014) noted that there was little consistency across studies in the recording of the amount or length of drug use, pointing out that many findings need to be considered with caution. Thus, it is difficult to study single dependencies empirically and the practical clinical relevance of doing so is questionable, as polydrug dependency is the more common clinical challenge.

As a result of these difficulties in recruiting individuals with single dependencies (excluding nicotine), our alcohol group was relatively small $(n=26)$, only providing sufficient power to detect moderate effect sizes (Smith et al. 2014). It may be more valuable for future investigations to consider all $\mathrm{AbD}$ participants as one group and investigate their different profiles that are not based on the substances used.

Another limitation of this study is that the groups differed on the measures of age, IQ and smoking status. Nevertheless, these variables were not found to correlate with any of the group differences identified in the present results, implying that they were not responsible for the differences found. It is perhaps surprising that there were such weak correlations between performance on the cognitive tasks and IQ. This is in line with some previous studies, but not all; for example a large recent study reported a significant correlation between 
IQ and delay discounting (de Wit et al. 2007). However it should be noted that other aspects of impulsivity were not related to IQ in that study, and furthermore the effect was observed in a much larger sample than that studied here and with a mean IQ markedly higher.

In addition, the high upper age range of participants within this study (65 years) may have introduced an age-related bias in impulsivity. There is substantial evidence of brain atrophy with older age, for example the rate of cortical atrophy increases to $0.35 \%$ a year after the age of 52 , compared to $0.12 \%$ in young adulthood, ventricle size expands at rate of $4.25 \%$ after 70 years compared to $0.43 \%$ in young adulthood, whilst the frontal lobes, which are involved in inhibitory control, show the steepest decline (for a review, see Dennis and Cabeza 2011). In addition, older adults show more compensation for poorer inhibitory control that declines with increasing age (Nielson et al. 2002), as well as poorer motor control (Levin et al. 2014). However, neither AbD group in the present study differed significantly in age from the control group (although the alcohol and polydrug groups differed) nor none of the variables showing group differences were correlated with age.

\section{Conclusion}

The present study suggests that the self-report measures used are more sensitive to detecting impulsivity in long-term abstinent individuals than the behavioural or neuronal measures. Our findings suggest the importance of developing behavioural measures that assess different aspects of impulsivity rather than simple motor response inhibition, alongside corresponding behavioural challenges to use in conjunction with fMRI. A complementary approach may be to reconsider the grouping of individuals in studies of dependence, with a shift of emphasis to cognitive endophenotypes rather than specific substances used. Such an approach would obviate the problem of categorising individuals with complicated drug use and dependence histories, and would have implications for optimising approaches to treatment and prevention based on cognitive profiles.

Acknowledgments This article presents independent research funded by the Medical Research Council as part of their addiction initiative (grant number G1000018). GSK kindly funded the functional and structural MRI scans that took place at Imperial College.

The research was carried out at the NIHR/Wellcome Trust Imperial Clinical Research Facility, the NIHR/Wellcome Trust Cambridge Research Facility and Clinical Trials Unit at Salford Royal NHS Foundation Trust, and is supported by the North West London, Eastern and Greater Manchester NIHR Clinical Research Networks. The views expressed are those of the author(s) and not necessarily those of the Medical Research Council, the NHS, the NIHR or the Department of Health.
We wish to thank research assistants Claire Whitelock, Heather Agyepong, Rania Christoforou and Natalie Cuzen for their help with data collection, MR physicist Rex Newbould and MR technician, Jonathan Howard for their assistance with MR acquisition and task set-up.

Recruitment partners- We wish to thank our recruitment partners; Imperial College Healthcare NHS Trust, Central and North West London NHS trust, Camden and Islington NHS trust, Cambridge University Hospitals NHS Foundation Trust, Norfolk and Suffolk NHS Foundation Trust, Cambridge and Peterborough NHS Foundation Trust, South Staffordshire and Shropshire NHS Foundation Trust, Manchester Mental Health NHS and Social Care Trust, Greater Manchester West NHS Foundation Trust, Pennine Care NHS Foundation Trust, Salford Royal NHS Foundation Trust, Addaction, Foundation 66 and CRI (Crime Reduction Initiative).

\section{Compliance with ethical standards}

Conflict of interest statement David Nutt is an advisor to British National Formulary, MRC, GMC, Dept of Health, is President of the European Brain Council, past President of the British Neuroscience Association and European College of Neuropsychopharmacology, chair of the Independent Scientific Committee on Drugs (UK), is a member of the International Centre for Science in Drug Policy, advisor to Swedish government on drug, alcohol and tobacco research, editor of the Journal of Psychopharmacology, sits on advisory Boards at Lundbeck, MSD, Nalpharm, Orexigen, Shire, has received speaking honoraria (in addition to above) from BMS/Otsuka, GSK, Lilly, Janssen, Servier, is a member of the Lundbeck International Neuroscience Foundation, has received grants or clinical trial payments from P1vital, MRC, NHS, Lundbeck, has share options with P1vital, has been expert witness in a number of legal cases relating to psychotropic drugs, and has edited/written 27 books - some purchased by pharma companies.

Trevor Robbins has research grants with Eli Lilly and Lundbeck, has received royalties from Cambridge Cognition (CANTAB), has received editorial honoraria from Springer Verlag, Elsevier, Society for Neuroscience; has performed educational lectures for Merck, Sharpe and Dohme and does consultancy work for Cambridge Cognition, Eli Lilly, Lundbeck, Teva and Shire Pharmaceuticals.

Bill Deakin currently advises or carries out research funded by Autifony, Sunovion, Lundbeck, AstraZeneca and Servier. All payment is to the University of Manchester.

Anne Lingford-Hughes has received speaking honoraria and research support from Lundbeck and research support from GSK.

Liam J Nestor is a Senior Research Scientist currently employed by GSK.

All other authors declared no conflict of interest.

Open Access This article is distributed under the terms of the Creative Commons Attribution 4.0 International License (http:// creativecommons.org/licenses/by/4.0/), which permits unrestricted use, distribution, and reproduction in any medium, provided you give appropriate credit to the original author(s) and the source, provide a link to the Creative Commons license, and indicate if changes were made.

\section{References}

Andersen SL, Teicher MH (2009) Desperately driven and no brakes: developmental stress exposure and subsequent risk for substance abuse. Neurosci Biobehav Rev 33(4):516-524

Aron AR et al (2003) Stop-signal inhibition disrupted by damage to right inferior frontal gyrus in humans. Nat Neurosci 6(2):115-116

Badiani A et al (2011) Opiate versus psychostimulant addiction: the differences do matter. Nat Rev Neurosci 12(11):685-700 
Bari A, Robbins TW (2013) Inhibition and impulsivity: behavioral and neural basis of response control. Prog Neurobiol 108:44-79

Bell RP, Garavan H, Foxe JJ (2014) Neural correlates of craving and impulsivity in abstinent former cocaine users: towards biomarkers of relapse risk. Neuropharmacology 85:461-470

Broos N et al (2012) The relationship between impulsive choice and impulsive action: a cross-species translational study. PLoS One 7(5):36781

Carver CS, White TL (1994) Behavioral inhibition, behavioral activation, and affective responses to impending reward and punishment: the BIS/BAS scales. J Pers Soc Psychol 67(2):319-333

Chambers CD, Garavan H, Bellgrove MA (2009) Insights into the neural basis of response inhibition from cognitive and clinical neuroscience. Neurosci Biobehav Rev 33(5):631-646

Clark L et al (2012) Striatal dopamine $\mathrm{D}_{2} / \mathrm{D}_{3}$ receptor binding in pathological gambling is correlated with mood-related impulsivity. NeuroImage 63(1):40-46

Comeau N, Stewart SH, Loba P (2001) The relations of trait anxiety, anxiety sensitivity, and sensation seeking to adolescents' motivations for alcohol, cigarette, and marijuana use. Addict Behav 26(6):803-825

Dalley JW, Everitt BJ, Robbins TW (2011) Impulsivity, compulsivity, and top-down cognitive control. Neuron 69(4):680-694

de Wit H (2009) REVIEW: impulsivity as a determinant and consequence of drug use: a review of underlying processes. Addict Biol 14(1):22-31

de Wit $\mathrm{H}$ et al (2007) IQ and non-planning impulsivity are independently associated with delay discounting in middle-aged adults. Personal Individ Differ 42(1):111-121

Dennis N, Cabeza R (2011) Neuroimaging of healthy cognitive aging. In: Craik F, Salthouse T (eds) The handbook of aging and cognition, 3rd edn. Psychology Press, New York

Diergaarde L et al (2008) Impulsive choice and impulsive action predict vulnerability to distinct stages of nicotine seeking in rats. Biol Psychiatry 63(3):301-308

Downes JJ et al (1989) Impaired extra-dimensional shift performance in medicated and unmedicated Parkinson's disease: evidence for a specific attentional dysfunction. Neuropsychologia 27(1112):1329-1343

Ersche KD, Sahakian BJ (2007) The neuropsychology of amphetamine and opiate dependence: implications for treatment. Neuropsychol Rev 17(3):317-336

Ersche KD et al (2010) Drug addiction endophenotypes: impulsive versus sensation-seeking personality traits. Biol Psychiatry 68(8):770-773

Ersche KD et al (2011) Abnormal structure of frontostriatal brain systems is associated with aspects of impulsivity and compulsivity in cocaine dependence. Brain: J Neurol 134(Pt 7):2013-2024

Evenden JL (1999) Varieties of impulsivity. Psychopharmacology 146(4):348-361

Evren C et al (2012) Relationship of relapse with impulsivity, novelty seeking and craving in male alcohol-dependent inpatients. Drug Alcohol Rev 31(1):81-90

Fernández-Serrano MJ, Pérez-García M, Verdejo-García A (2011) What are the specific vs. generalized effects of drugs of abuse on neuropsychological performance? Neurosci Biobehav Rev 35(3):377-406

Fernández-Serrano MJ et al (2012) Neuropsychological profiling of impulsivity and compulsivity in cocaine dependent individuals. Psychopharmacology 219(2):673-683

Foa EB et al (2002) The obsessive-compulsive inventory: development and validation of a short version. Psychol Assess 14(4):485-496

Forman SD et al (2004) Opiate addicts lack error-dependent activation of rostral anterior cingulate. Biol Psychiatry 55(5):531-537

$\mathrm{Fu} \mathrm{L}$ et al (2008) Impaired response inhibition function in abstinent heroin dependents: an fMRI study. Neurosci Lett 438(3):322-326
Garavan $\mathrm{H}$ et al (2006) Individual differences in the functional neuroanatomy of inhibitory control. Brain Res 1105(1):130-142

Gillespie NA et al (2012) Two-part random effects growth modeling to identify risks associated with alcohol and cannabis initiation, initial average use and changes in drug consumption in a sample of adult, male twins. Drug Alcohol Depend 123(1-3):220-228

Harty SC et al (2011) Impulsive choice, as measured in a delay discounting paradigm, remains stable after chronic heroin administration. Pharmacol Biochem Behav 98(3):337-340

Hester R, Garavan H (2004) Executive dysfunction in cocaine addiction: evidence for discordant frontal, cingulate, and cerebellar activity. J Neurosci: Off J Soc Neurosci 24(49):11017-11022

Hopwood CJ et al (2011) Pathological personality traits among patients with absent, current, and remitted substance use disorders. Addict Behav 36(11):1087-1090

Kaufman JN et al (2003) Cingulate hypoactivity in cocaine users during a GO-NOGO task as revealed by event-related functional magnetic resonance imaging. J Neurosci 23(21):7839-7843

Kelly TH et al (2006) Individual differences in drug abuse vulnerability: d-amphetamine and sensation-seeking status. Psychopharmacology 189(1):17-25

Kirby KN (2000) Instructions for inferring discount rates from choices between immediate and delayed rewards. Williamstown, MA, USA

Kirby KN, Maraković NN (1996) Delay-discounting probabilistic rewards: rates decrease as amounts increase. Psychon Bull Rev 3(1): 100-104

Kirby KN, Petry NM, Bickel WK (1999) Heroin addicts have higher discount rates for delayed rewards than non-drug-using controls. J Exp Psychol Gen 128(1):78-87

Koob GF, Le Moal M (2001) Drug addiction, dysregulation of reward, and allostasis. Neuropsychopharmacology 24(2):97-129

Levin $\mathrm{O}$ et al (2014) Aging and motor inhibition: a converging perspective provided by brain stimulation and imaging approaches. Neurosci Biobehav Rev 43:100-117

Li CR et al (2008) Neural correlates of impulse control during stop signal inhibition in cocaine-dependent men. Neuropsychopharmacol: Off Publ Am Coll Neuropsychopharmacol 33(8):1798-1806

Li C-SR et al (2009) Altered impulse control in alcohol dependence: neural measures of stop signal performance. Alcohol Clin Exp Res 33(4):740-750

Liao DL et al (2014) Cognitive control in opioid dependence and methadone maintenance treatment. PLoS One 9(4):e94589, H. de Wit, ed

MacKillop J, Kahler CW (2009) Delayed reward discounting predicts treatment response for heavy drinkers receiving smoking cessation treatment. Drug Alcohol Depend 104(3):197-203

Mahoney JJ et al (2015) A comparison of impulsivity, depressive symptoms, lifetime stress and sensation seeking in healthy controls versus participants with cocaine or methamphetamine use disorders. J Psychopharmacol (Oxford, England) 29(1):50-56

Marusich JA et al (2011) A multivariate assessment of individual differences in sensation seeking and impulsivity as predictors of amphetamine self-administration and prefrontal dopamine function in rats. Exp Clin Psychopharmacol 19(4):275-284

McCown W (1988) Multi-impulsive personality-disorder and multiple substance abuse: evidence from members of self-help groups. Br J Addict 83(4):431-432

Moeller FG et al (2001) The impact of impulsivity on cocaine use and retention in treatment. J Subst Abus Treat 21(4):193-198

Morie KP et al (2014) Intact inhibitory control processes in abstinent drug abusers (II): a high-density electrical mapping study in former cocaine and heroin addicts. Neuropharmacology 82:151-160

Naim-Feil J et al (2014) Neurocognitive deficits, craving, and abstinence among alcohol-dependent individuals following detoxification. Arch Clin Neuropsychol: Off J Natl Acad Neuropsychol 29(1):26-37 
Nielson KA, Langenecker SA, Garavan H (2002) Differences in the functional neuroanatomy of inhibitory control across the adult life span. Psychol Aging 17(1):56-71

Noël X et al (2011) Neurocognitive determinants of novelty and sensation-seeking in individuals with alcoholism. Alcohol Alcohol (Oxford, Oxfordshire) 46(4):407-415

Nutt DJ, King LA, Phillips LD (2010) Drug harms in the UK: a multicriteria decision analysis. Lancet 376(9752):1558-1565

Passetti F et al (2008) Neuropsychological predictors of clinical outcome in opiate addiction. Drug Alcohol Depend 94(1-3):82-91

Paterson LM et al (2015) The Imperial College Cambridge Manchester (ICCAM) platform study: an experimental medicine platform for evaluating new drugs for relapse prevention in addiction. Part A: study description. J Psychopharmacol (Oxford, England) 29(9): 943-960

Patkar AA et al (2004) Pre-treatment measures of impulsivity, aggression and sensation seeking are associated with treatment outcome for African-American cocaine-dependent patients. J Addict Dis 23(2): $109-122$

Patton JH, Stanford MS, Barratt ES (1995) Factor structure of the Barratt impulsiveness scale. J Clin Psychol 51(6):768-774

Perry JL, Carroll ME (2008) The role of impulsive behavior in drug abuse. Psychopharmacology 200(1):1-26

Schippers MC et al (2012) Unidirectional relationship between heroin self-administration and impulsive decision-making in rats. Psychopharmacology 219(2):443-452

Schulte MHJ et al (2014) Recovery of neurocognitive functions following sustained abstinence after substance dependence and implications for treatment. Clin Psychol Rev 34(7):531-550

Semple SJ et al (2005) Impulsivity and methamphetamine use. J Subst Abus Treat 29(2):85-93

Shin SH, Hong HG, Jeon S-M (2012) Personality and alcohol use: the role of impulsivity. Addict Behav 37(1):102-107

Shin SH, Chung Y, Jeon S-M (2013) Impulsivity and substance use in young adulthood. Am J Addict Am Acad Psychiatr Alcohol Addict 22(1):39-45

Simmonds DJ, Pekar JJ, Mostofsky SH (2008) Meta-analysis of Go/Nogo tasks demonstrating that fMRI activation associated with response inhibition is task-dependent. Neuropsychologia 46(1):224-232

Sinha R (2011) New findings on biological factors predicting addiction relapse vulnerability. Curr Psychiatry Rep 13(5):398-405

Sjoerds Z et al (2014) Response inhibition in alcohol-dependent patients and patients with depression/anxiety: a functional magnetic resonance imaging study. Psychol Med 44(8):1713-1725

Smith JL et al. (2014) Deficits in behavioural inhibition in substance abuse and addiction: a meta-analysis. Drug Alcohol Depend
Stavro K, Pelletier J, Potvin S (2013) Widespread and sustained cognitive deficits in alcoholism: a meta-analysis. Addict Biol 18(2): 203-213

Stevens L et al (2013) Disadvantageous decision-making as a predictor of drop-out among cocaine-dependent individuals in long-term residential treatment. Frontiers Psychiatry 4:149

Stevens L et al (2014) Impulsivity as a vulnerability factor for poor addiction treatment outcomes: a review of neurocognitive findings among individuals with substance use disorders. J Subst Abus Treat 47(1):58-72

Stevens L et al (2015) Impulsive choice predicts short-term relapse in substance-dependent individuals attending an in-patient detoxification programme. Psychol Med 45(10):1-11

Stoops WW et al (2007) The reinforcing, subject-rated, performance, and cardiovascular effects of d-amphetamine: influence of sensationseeking status. Addict Behav 32(6): 1177-1188

Sullivan EV et al (2000) Longitudinal changes in cognition, gait, and balance in abstinent and relapsed alcoholic men: relationships to changes in brain structure. Neuropsychology 14(2):178-188

Torres A et al (2013) Emotional and non-emotional pathways to impulsive behavior and addiction. Front Hum Neurosci 7:43

Vassileva J et al (2014) Heroin and amphetamine users display opposite relationships between trait and neurobehavioral dimensions of impulsivity. Addict Behav 39(3):652-659

Verdejo-García A, Lawrence AJ, Clark L (2008) Impulsivity as a vulnerability marker for substance-use disorders: review of findings from high-risk research, problem gamblers and genetic association studies. Neurosci Biobehav Rev 32(4):777-810

Volkow ND et al (2011) Addiction: beyond dopamine reward circuitry. Proc Natl Acad Sci 108(37):15037-15042

von Diemen L et al (2008) Impulsivity, age of first alcohol use and substance use disorders among male adolescents: a population based case-control study. Addict (Abingdon, England) 103(7):1198-1205

Washio Y et al (2011) Delay discounting is associated with treatment response among cocaine-dependent outpatients. Exp Clin Psychopharmacol 19(3):243-248

Whelan R et al (2014) Neuropsychosocial profiles of current and future adolescent alcohol misusers. Nature 512(7513):185-189

Whiteside SP, Lynam DR (2003) Understanding the role of impulsivity and externalizing psychopathology in alcohol abuse: application of the UPPS impulsive behavior scale. Exp Clin Psychopharmacol 11(3):210-217

Woicik PA et al (2009) The substance use risk profile scale: a scale measuring traits linked to reinforcement-specific substance use profiles. Addict Behav 34(12):1042-1055

Zuckerman M (2007) Sensation seeking and substance use and abuse: smoking, drinking, and drugs, Sensation seeking and risky behavior. American Psychological Association, Washington, DC, pp 107-143 